\title{
Non-human primates in medical research and drug development: a critical review
}

\author{
JARROD BAILEY* \\ School of Population and Health Sciences, University of Newcastle upon Tyne, NE2 4AA, UK
}

Received 20 March 2005; accepted 20 July 2005

\begin{abstract}
There is much current debate surrounding the use of non-human primates (NHPs) in medical research and drug development. This review, stimulated by calls for evidence from UK-based inquiries into NHP research, takes a critical view in order to provide some important balance against papers supporting NHP research and calling for it to be expanded. We show that there is a paucity of evidence to demonstrate the positive contribution or successful translation of NHP research to human medicine, that there is a great deal of often overlooked data showing NHP research to be irrelevant, unnecessary, even hazardous to human health and to have little or no predictive value or application to human medicine. We briefly discuss the reasons why this may be so, reflect upon the consequences for future medical progress and, on the basis of our findings, suggest a more scientifically robust and promising way forward.
\end{abstract}

Keywords: Primates; NHP; monkeys; animal.

\section{INTRODUCTION}

Such is the current level of controversy surrounding the issue of NHP experimentation, that in recent years a number of inquiries have taken place into the practice. For example, in December 2002 the Animal Procedures Committee (APC) in the UK published a report containing recommendations for its future (Animal Procedures Committee, 2002); earlier this year the committee held and published the proceedings of a subsequent stakeholders' forum, for which it has invited comments (Home Office Primates Stakeholder Forum, 2004). Also, in March 2005 a new committee was set up to examine the scientific basis of NHP experimentation, for which submissions of evidence were invited (http://www.nhpstudy.com/call_for_evidence.pdf). This is to be warmly encouraged; only by objectively questioning and evaluating scientific methodologies without bias can we hope to ensure that it is of optimal efficacy and likely to achieve

*E-mail: jarrod.bailey@ncl.ac.uk 
its long-term goals. At the same time, we must be conscious of who commissions, oversees and publishes such reports: objectivity is often compromised by vested interests. Nevertheless, such inquiries at least provide an opportunity for the airing of views across the whole spectrum, and some assessment is better than none at all. It is fair to say that, were it not for these inquiries, it would be left to NHP research reports in the scientific literature to shape scientists' views. Arguably, this area of endeavour suffers more than most from the 'positive bias' that affects the publication policies of many journals, perhaps due to the attention that is focussed on it. In taking a critical, yet still balanced and comprehensive stance in this review, which is drawn from submissions of evidence to the above two inquiries, we hope to redress this balance to some degree while at all times maintaining a scientific and factual attitude to stimulate debate on the matter.

Many papers reveal findings that, while constituting interesting contributions to the scientific knowledge-base, are not relevant or applicable to human medicine. To be of use and scientifically justifiable they must be predictive of the human situation, providing information that is capable of translation to the clinic. Many studies provide only fortuitous 'experimental confirmation' of previously known human clinical data, or are championed when they correlate retrospectively with human studies. In both of these cases, or when the reported studies have demonstrated fundamental inter-species differences, the authors' conclusions tend towards crucial 'further work' based, naturally, on more experiments involving NHPs. We suggest that a more critical approach would be more beneficial to medicine, and we provide a brief outline of such an approach here.

\section{USE OF NON-HUMAN PRIMATES IN DRUG DEVELOPMENT/TOXICOLOGY}

The major use of NHPs remains as the 'second species' of animal in pre-clinical safety assessment of new drugs. In spite of such testing, the attrition rate of drugs entering human clinical trials is $92 \%$ (Harding, 2004). Many drugs that do pass the clinical trial phase to reach the market place have a questionable safety profile: adverse drug reactions (ADRs) remain the fourth biggest killer in the western world, despite apparent safety in animal (often NHP) models (Lazaru et al., 1998). Important examples include:

- Hormone Replacement Therapy — prescribed to millions of women, and thought to protect against heart disease and stroke on the basis of NHP experiments, HRT is now known to increase the risk of these diseases, as well as breast cancer in humans. The Chairman of the German Commission on the Safety of Medicines described HRT as 'the new Thalidomide'. It has caused up to 20000 cases of breast cancer over the past decade in Britain alone (Gray, 2003).

- AIDSVAX - after successful results of this HIV vaccine in chimpanzees, it failed to protect any of the 8000 human volunteers in clinical trials.

- Teratology (risk of birth defects) - in the wake of Thalidomide, animals, often NHPs, have been used to assess the risk of foetal damage during pregnancy as 
a result of exposure to drugs and other chemicals. Results from NHPs correlate with known human teratogens only $50 \%$ of the time, less even than results from more evolutionarily distant species such as rats, hamsters and ferrets (Schardein, 1993; Bailey et al., 2005). Aspirin, for example, is teratogenic in monkeys though not in humans.

- Asthma - Isoprenaline doses, derived from animal data, were too high for humans and killed thousands of people; mainly children. An attempt to replicate this in NHPs was completely unsuccessful (Carson et al., 1971).

- Opren (for arthritis) killed 61 people and harmed over 3500, despite showing no problems in NHPs (Eason et al., 1990).

- Flosint (another arthritis drug) caused hundreds of severe ADRs and several deaths, despite being well tolerated in NHPs (Mann, 1984).

- Carbenoxalone (for gastric ulcers) caused heart failure, which could not be replicated in NHPs (Eason et al., 1990).

- Amrinone (for heart failure) caused severe haemorrhaging in $20 \%$ of long-term patients, despite showing no warning signs in NHPs (Eason et al., 1987).

These statistics and examples are of no surprise when one considers comprehensive studies of comparative drug toxicology, which have revealed levels of discordance between results from animals and humans of between 67 and 96\% (Lumley and Walker, 1990; Spriet-Pourra and Auriche, 1994). These studies show that animal (or NHP) based toxicology is not predictive of human response; providing correct predictions less often than a coin toss.

Among scientists in academia and in the pharmaceutical industry itself, the inability of animal-based pre-clinical models (including NHPs) to assess human ADMET properties (how a drug is Absorbed, Distributed, Metabolised and Eliminated, and its Toxicological properties) has been acknowledged for decades:

- Dr. Brimblecombe of Smith Kline and French commented in 1981, '... the present tests are well known to us but that does not make them good. There may be better tests around, but we have no incentive whatsoever to look for them at the moment. In fact, quite the reverse [because of the fear that regulatory agencies will require the new tests in addition to the current ones] (Brimblecombe, 1981)'.

- Professor Caldwell of St. Mary's Hospital Medical School, London observed, 'It has been obvious for some time that there is generally no evolutionary basis behind the particular-metabolizing ability of a particular species. Indeed, among rodents and primates, zoologically closely related species exhibit markedly different patterns of metabolism (Caldwell, 1992)'.

- William Bains, chief scientific officer of Amedis Pharmaceuticals (Cambridge, UK) estimates that $50 \%$ of all drugs in development fail to progress to the market because of problems associated with ADMET and 50\% of all drugs that do make it to market have problems associated with ADMET (Hodgson, 2001).

- Drs Palfreyman, Charles and Blander in 'Drug Discovery World' observed that 'One of the major challenges facing the drug discovery community is the 
limitation and poor predictability of animal-based strategies ... many drugs have failed in later stages of development because the animal data were poor predictors of efficacy in the human subject .... One of the overriding interests of the pharmaceutical and biotechnologies industry is to create alternative development strategies that are less reliant on poor animal predictor models of human disease ...' (Palfreyman et al., 2002).

Marmosets are often the 'second species' of choice — not due to any outstanding scientific properties or especially predictive qualities but because they are small, cheap and relatively easy to house and breed (Smith et al., 2001). In fact, the validity of NHP use in this way has been questioned recently in several quarters:

- The Toxicology Working Group of the House of Lords Select Committee on Animals in Scientific Procedures stated 'the formulaic use of two species in safety testing is not a scientifically justifiable practice, but rather an acknowledgement of the problem of species differences in extrapolating the results of animal tests to predict effects in humans', and 'the reliability and relevance of all existing animal tests should be reviewed as a matter of urgency' (House of Lords Select Committee on Animals in Scientific Procedures, 2002).

- The Boyd Group concluded that 'any use of NHPs in research and testing requires very strong justification' and recommended that the APC 'examine retrospectively any application or licence to use non-human primates, with a view to informing future ethical judgements' (Boyd Group papers, 2002).

- The Nuffield Council on Bioethics concluded that 'there is a need for continuing review of the scientific case for using animals in research and testing. It is axiomatic that any such use should be accompanied by active and critical reflection on the validity and relevance of the models and research studies' (Nuffield Council on Bioethics, 2005).

In addition, the Animal Procedures Committee produced a series of wide-ranging recommendations and associated observations following their aforementioned inquiry (Animal Procedures Committee, 2002). Many of these are briefly summarised below, along with some relevant thoughts and criticisms:

- "The development and implementation of non-animal alternatives to replace the use of non-human primates must be accepted within industry and the international regulatory arena as a high priority goal".

The committee are to be applauded for this recommendation, and also their insistence that industry 'can do better', that non-animal alternatives might constitute 'better science' and their conclusion that there are major advantages to be gained from the replacement of NHPs.

However, they also concluded that there are 'no quick wins in sight', suggesting a concentration of scientific effort in the near future on reduction and refinement in preference to replacement, and that gains in the former ' $2 \mathrm{Rs}$ ' are more likely in the short term than gains in the latter. These 'gains' might be true if the focus is restricted to animal numbers and/or suffering, but any gain with regard to human 
health is questionable at best. If we acknowledge that 'better science' is a tangible advantage of the acceptance, development and implementation of non-animal alternatives, then it is surely counter-productive to divert attention and resources away from replacement in favour of reduction and refinement. There is substantial and increasing evidence that animal models are not scientifically justifiable, and therefore their refinement and reduction is highly unlikely to translate to any benefit for human medicine.

In the opinion of a growing number of scientists, replacement is the only valid way forward and a multitude of technologies has already been developed to facilitate this. When used together, these are clearly more predictive of the human situation than the animal (and often NHP) models that are current standard practice. For example, recent advances with microarray technology allied to computer learning methods have shown huge promise in toxicity testing. One group developing this is SimuGen (Cambridge, UK), who have stated a view shared by many, "The proof is overwhelming that it will work ... the long term plan is to replace animal testing ... the technology is already there, but drugs regulators are very strict as to what is appropriate and safe."

- "We believe it is essential to instigate a detailed examination of regulatory policies on species selection in toxicity testing ..."

An examination of this type, in conjunction with full disclosure of the policies of regulatory authorities, would be of real scientific value. It has been argued long and hard by thousands of concerned scientific and medical professionals that it is not scientifically possible to reliably extrapolate findings from one species to another. This conviction was echoed by the Toxicology Working Group of the House of Lords select committee on Animals in Scientific Procedures (House of Lords Select Committee on Animals in Scientific Procedures, 2002), whose comments are detailed above, as are observations along similar lines from the Nuffield Council on Bioethics (Nuffield Council on Bioethics, 2005).

- "The forum understood that the thinking behind this APC recommendation was that pharmaceutical companies often used primates for reasons other than scientific necessity".

The evidence for this view is that the scientific necessity of NHP use has never been demonstrated by those that advocate it. In fact, in addition to myriad examples illustrating the folly of NHP use, there is anecdotal evidence to embarrass those advocates. For example, Dr. Robert Ruffalo, President of R\&D at Wyeth, cited at an international conference in March 2005 an example involving the pneumococcal vaccine Prevnar. Successfully used in over 30 million American children since the year 2000, the human epidemiological data were of no interest to Japanese regulatory authorities, who required new NHP data instead. Indeed, the fact that human trials often take place concurrently with or even prior to animal toxicology is evidence that animal tests are performed for regulatory, rather than scientific, reasons. Further evidence is provided by the Association of the British 
Pharmaceutical Industry in a paper entitled 'The selection of marmoset monkeys in pharmaceutical toxicology' (Smith et al., 2001) which documents considerations of cost, practicality and convenience as reasons for their use.

- "There may be a transient, but significant, increase in animal numbers whilst information to inform discussion of suitability was gathered on the basis of comparative studies".

After decades of animal-based toxicology, there is more than enough data with which to perform comparative studies, such as those mentioned above (Lumley and Walker, 1990; Spriet-Pourra and Auriche, 1994). Last year a review of the evidence that animals are reliable predictors of toxic effects in humans found the evidence to be 'fragmentary', pointing to 'significant over- and under-prediction of adverse effects from animal studies that varies with the particular organ or system'. Furthermore, it showed the false positive rate to be astonishingly high: even in NHPs it exceeded the success rate in two-thirds of the forms of toxicity studied (Greaves, 2004).

- "The Home Office should insist that a full range of in vitro toxicokinetic/metabolism screening be done before ... the selection of a second (non-rodent) species for drug safety evaluation".

The committee's decision that a full range of in vitro ADMET (how a drug is Absorbed, Distributed, Metabolised and Eliminated, and its Toxicological properties) studies be made obligatory during the course of drug development is laudable. However, there is a strong argument that the range, diversity, relevance and performance of batteries of powerful in vitro, in silico, human ex vivo, microdosing and other studies negate any data that can be provided from animal models; it follows that using data that is directly relevant to humans as the basis for the choice of a poorly predictive animal model is of questionable scientific merit. Scientific justification for the use of a particular animal species against human-specific methods, rather than simply against another animal species, would be far more apposite. Scientists in academia and in the pharmaceutical industry itself have acknowledged for decades the inability of animal-based pre-clinical models (including NHPs) to assess human ADMET properties, as discussed above.

- "It was recognized and acknowledged that tremendous progress has been made in identifying and progressing appropriate preliminary studies and that industry deserves credit".

Credit must be given to those who have conceived and developed many of the studies and tests that provide more pertinent and reliable data to aid in the prediction of human drug response. In this way, drugs are being and have been developed precluding any reliance on animal pre-clinical data, at least until regulatory guidelines demand it: examples include anti-HIV protease-inhibitor and nucleoside-analogue drugs (DeVita et al., 1992; Mitsuya et al., 1985; Seelmeier et al., 1988; Soudeyns et al., 1991; Roberts et al., 1990; Vacca et al., 1994), some of which are known to have ADMET properties that vary wildly between 
animal species and that can be particularly dissimilar in NHPs (Chiba et al., 2000); Novartis' leukaemia drug Glivec that was designed and developed in vitro (Druker and Lydon, 2000; Druker et al., 1996; Geissler et al., 1992; Lydon et al., 1990), and several drugs in the development pipeline from the company Pharmagene, one of which, for cystic fibrosis (PGN0052) is in late-phase clinical trials (http://www.pharmagene.com/).

- "The availability of animal tissue for comparative in vitro studies should be improved and we urge the pharmaceutical industry, the Home Office and ERPs to promote in-house tissue sharing and further promote tissue banks".

The committee's encouragement of the use of human tissues, which are readily available through numerous channels such as the UK Human Tissue Bank (http://www.ukhtb.org), members of the British Association for Tissue Banking (http://www.batb.org.uk/), and various commercial suppliers, will have a far more significant positive scientific impact.

- "The use of highly sensitive analytical methods to provide human pharmacokinetic data should be further developed and the resources provided to move technologies from the research phase to the stage where they can be routinely used. Early ultra-low dose studies in human volunteers should be encouraged".

There can be no argument with this recommendation; even the Food and Drug Administration (FDA) has accepted that 'the tools of the last century are being used to develop the drugs of the 21st (http://www.fda.gov/oc/initiatives/criticalpath/ whitepaper.html).

Concerns were raised, however, about limitations of these techniques; about increased animal use in later stages of development if more drugs pass the screening process; about the expense, infancy and limited availability of microdosing; and about predictability and human safety, and these issues must be addressed.

All scientific techniques used in drug development have limitations; the critical question to ask is which combination of them provide the most relevant, predictive and reliable data with which to proceed to clinical trials. Currently the drug development process is heavily animal-based, relying on methods that correlate poorly with the human response, which is largely responsible for the fact that over $90 \%$ of drug candidates fail during human clinical trials. As discussed earlier, a comprehensive battery of human-specific tests presents us with the shortest 'leap of faith' possible when proceeding to the clinical phase of development. To continue using animal (often NHP) models that have never been validated and with all their limitations, while insisting upon extreme validation criteria for alternative methods is unscientific.

One of the objections of the APC forum, that more animals would be used in pre-clinical development if prior human-based screening and discovery methods were adopted, is not a valid reason to resist them even if this were true. More pertinent human-based screening and discovery methods would help to deliver the 'fail early, fail cheap' holy grail of the pharmaceutical industry, leading to the 
progression of fewer compounds, but better compounds, to clinical trials where the real expense lies. If drug companies extended this human-centric approach to the development phase, as they could and should, they may well find such compounds have a lower chance of adding to the adverse drug reaction (ADR) statistics that are now responsible for harming and killing a number of people beaten only by cancer, heart disease and stroke. With regard to the 'predictability' and 'human safety' concerns of the forum, moving away from animal-based methods is surely critical to address these.

Events have shown the forum's concerns about microdosing to be unfounded. Even if expense were a genuine issue, it ought to be a pharmaceutical company's foremost concern that any drug they develop is launched with the best human efficacy and safety data available. It is perhaps timely here to reiterate the burden of expense in the pharmaceutical industry; $R \& D$ costs are typically one-quarter of the advertising and marketing budget, and only $15 \%$ of this is for pre-clinical studies.

So if expense is not a real issue, is the relative infancy of the technique itself? Notably, microdosing was recently rigorously tested with five drugs known for their difficult pharmacokinetic properties, in a trial designed for the technique to be unlikely to succeed. This 'CREAM' (Consortium for Resourcing and Evaluating AMS Microdosing) trial was pronounced a real success (http://www.xceleron.co.uk/ metadot/index.pl?id=2224\&isa=Newsitem\&op=show), and the technique has also been given a seal of approval by the FDA (April 2005) and the European Agency for the Evaluation of Medicinal Products (EMEA, January 2003).

- "The use of primates in the safety assessment of pharmaceuticals can clearly only be justified under current UK legislation if the data obtained are both valid (relevant for humans) and necessary in order for a safety assessment to be made. Validity and necessity should be continuously monitored by retrospective comparison of test data with clinical experience, and the need for studies specifically on primates should be critically assessed before tests are carried out".

Validity and relevance for humans are surely pre-requisites for any techniques used in the safety assessment of drugs. It is incredible, therefore, that so little analysis of these aspects has been carried out for what is an intrinsic part of drug development. Earlier in this document were summarised some of the studies done to date. In addition, the Olson study cited by the APC (Olson et al., 2000) found that if a side effect occurred in humans, there was an animal that it also occurred in about $70 \%$ of the time. Of course in retrospect it is simple to find an animal from all available species used in toxicology that replicates the human condition. Prediction, knowing in advance which species will be suggestive of the human condition is another matter, and this is where the animal model fails. The Greaves study (Greaves et al., 2004) cited earlier revealed an alarmingly high false positive rate. It also does not stand up to statistical scrutiny: much of the evidence supporting the predictive nature of animal toxicity was derived from an earlier study (Schein et al., 1970) with incorrectly calculated false positive and false negative rates (Personal 
communication from professional statistician). Correctly manipulated statistics actually demonstrate that dogs and monkeys, even when the results are taken in tandem, are no more predictive of human toxicity than a coin toss; they give no weight of evidence to prior odds that a particular compound is toxic to humans, and they actually show that toxicity results for animals (including NHPs) and humans are independent of one another.

It is therefore already abundantly clear, despite the relative paucity of studies that have examined the plethora of toxicological data available, that animal studies in pre-clinical drug development are neither valid for nor relevant to humans. Often, data from NHPs, our closest genetic relatives, correlates with human data less than that from other more distant species. Even Ralph Heywood, former director of Huntingdon Research Centre, stated 'the best guess for the correlation of adverse reactions in man and animal toxicity data is somewhere between 5 and $25 \%$ '.

- "The predictive value of data from primate studies should be investigated by comparing the results of pre-clinical and clinical studies on drugs that have progressed to clinical use".

"Such a comparison could provide an indicator of study validity with respect to materials that subsequently make it into humans".

This information would be essential in helping to determine the true predictive value of NHPs as a pre-clinical model, but would of course only provide limited information: many 'False Positive' compounds would be missed because they did not progress to clinical trials. This endeavour, however, would serve to confirm beyond doubt whether the use of NHPs (and indeed other animals) in pre-clinical toxicology is a scientifically justifiable undertaking. More evidence of this type would hasten the move towards the most effective drug development methodologies, translating to safer and more effective drugs for society.

The concerns of certain members of the forum in this instance were insubstantial:

- "Simply acquiring the data would not provide sufficient information to critically appraise decisions on whether or not to use primates".

Acquiring these data is essential, and is better than doing nothing at all to scientifically appraise primate use. Put simply, NHP data is either predictive (as it should be) or not. If this data shows a lack of correlation, then the data is not predictive, and NHP use is not justifiable.

- "An informed choice of second species would have been made before the data was generated, and it would be difficult to determine what the findings might have been if another species had been used".

This is a salient point in the scientific argument against the use of NHPs and animals in general; one cannot determine what the findings might have been for another species because it is impossible to extrapolate such data between them; this includes humans.

- "As clinical studies are not designed to produce or evaluate toxic effects in humans, correlation of findings related to toxicity would not be expected". 
This is an extraordinary claim: clinical studies may not be designed to produce toxic effects, but one of their accepted and important roles is most certainly to evaluate those that do occur. Many ADRs are detected during clinical trials, and these can be retrospectively compared with those arising in pre-clinical NHP models: correlation is then possible.

If, as claimed above, clinical trials are not designed to evaluate toxic effects then it must be assumed that this is one of the roles of pre-clinical animal models. Once again, the lack of predictivity of animal toxicology, the numbers of reported deaths in clinical trials despite extensive animal data (Marshall, 2000), and the increasing rates of morbidity and mortality from ADRs must be considered.

- "As the findings in the second species can prevent materials reaching clinical trials, and prior to this many compounds will already have been screened out based on other species' toxicity/in-vitro/in-silico, such a study would also not be informative about the decisions not to proceed to humans taken on the basis of the findings in the second species".

Findings in the first species do not always signal the end of the drug assessment process, and so important comparative information between animal species could be gathered. This information could further be used to assess the correlation and predictive nature of the animal models with regard to humans, for example when compounds proceed to clinical trial despite some adverse findings in the animal models, or in cases where compounds 'pass' the animal-based tests but fail in clinical trials.

- "The design and sequence of pre-clinical safety studies needs to be reviewed. We ask the Home Office to consider whether measures need to be taken to prevent overlap of rodent and non-rodent studies, actively discouraging any simultaneous testing in rodents and primates in order to shorten the time course of drug development".

A review of the practices involved in pre-clinical studies is long overdue, and absolutely essential in light of the current level and rate of growth of ADRs. However, the focus should not be on a simple alteration of a protocol that tacitly accepts the worth of animal (including NHP) models: the increasing amount of negative evidence with regard to the scientific validity of these, coupled with technological advances that have presented many better alternatives, surely demand that nothing short of a complete overhaul will suffice.

- "However, tests on rodent species can fail to identify toxic effects (which will more accurately predict the effect on man) found in primates".

Tests on rodent species cannot predict toxic effects found in NHPs; they can fail to correlate with them, and often do. It follows that there is also a poor correlation between rodents and humans, and of course between NHPs and humans. Such poor correlation is found between all species, and even between different strains of the same species, and is responsible for the complete lack of predictivity between species. Statistics do not support the statement made above by the forum that toxic 
effects found in NHPs more accurately predict the effect on man; therefore, the earlier statements above concerning a complete overhaul of pre-clinical studies, the support for this from the House of Lords Select Committee on Animals in Scientific Procedures (2002) and the Nuffield Council on Bioethics (2005), and other statistics and quotations above that constitute much of the evidence base for this, take on more importance.

- "Species selection is important for both ethical and scientific reasons; and it must be made on a sound scientific basis".

"Since pharmaceuticals are being developed for use in the human, the most appropriate species will be the one that most closely resembles the human".

"... the Primates sub-committee considers for the reasons set out in chapter 3 of the report that the choice is currently insufficiently justified. We consider that this problem is significant...".

Taking into account the arguments detailed above, the only species that is scientifically justifiable for selection is Homo sapiens. The argument for animal-based preclinical testing has always been the necessity of testing in a 'whole system'. When the available whole systems are quite clearly, in every respect, the 'wrong systems', this argument fails to hold. This can be illustrated by a revision of the small number of examples listed earlier, of how species differences between humans and NHPs have resulted in human harm.

\section{NON-HUMAN PRIMATES IN HUMAN DISEASE RESEARCH}

NHP research on the three leading killers; heart disease, cancer and stroke, has failed to yield significant insights about the diseases in humans, or to produce drugs to treat them. The scientific literature is replete with examples of NHP data confounding human disease research by conflicting with known human data or by leading research up 'blind alleys', or even by causing human harm when translated to the clinic. Looking briefly at some of the major areas of NHP use:

- Stroke occurs as a result of atherosclerosis, but atherosclerotic plaques do not form in NHPs. Artificial induction of stroke-like situations in NHPs have served as a model of stroke research for decades, despite critical physiological differences such as collateral blood flow. Significant species-specific and even strain-specific differences in response to ischaemic injury exist (Huang et al., 2000), and despite dozens of candidates, not a single effective therapy has been successful in humans, despite efficacy in NHPs (Neff, 1989; Wiebers et al., 1990a, b).

- Parkinson's Disease (PD) has been studied using neurotoxic chemicals to induce superficial PD-like symptoms, predominantly in marmosets and macaques. Fundamental differences in the onset, type, and persistence of symptoms exist in all models, in addition to physiological differences such as the absence in NHPs of 
Lewy Bodies. Species differences are known to play a role in the clinical expression as well as in the cellular specificity of the lesions; for example, striatal degeneration in humans is frequently associated with dyskinesia, whereas striatal excitotoxic lesions alone are not sufficient to induce dyskinesia or chorea in NHPs. Also, the time course of nerve cell degeneration, which normally evolves over several years in neurodegenerative diseases in humans, is for practical reasons replaced by a much shorter period of time in NHP models (Hantraye, 1998). The major breakthroughs for PD have been via epidemiology, clinical studies, genetic research, human tissue studies and autopsies. Space precludes detail here, but these have resulted in the discovery that levodopa crosses the blood-brain barrier then converts into dopamine, that its efficacy in treatment of PD patients diminishes due to progressive degeneration of the $\mathrm{D} 3$ dopamine receptor, and that there seem to be a number of genetic and environmental predispositions/causes. Additionally, deep-brain stimulation (DBS), often claimed to have been developed through critical NHP experiments, was actually discovered serendipitously in a human patient and owes nothing to NHPs for its advancement (Benabid et al., 1987, 1989).

- Alzheimer's disease $(A D)$ is another human ailment for which progress has been confounded and hampered by animal (including NHP) research. Many scientists have spent years trying to create a 'good' AD animal model; not only has this been a failure (Conner and Tuszynski, 2000; Lindholm, 1997; Snowdon et al., 1997), but very little progress has been made in understanding the various pathologies associated with the disease. For example, mouse experiments suggested that tau protein was unlikely to be of any importance, though this is now known to be highly species-specific (Guela et al., 1998), and plaques and tangles in the brain are the hallmark of Alzheimer's disease in humans but not in monkeys (St. George-Hyslop and Westaway, 1999). Human clinical research, epidemiological studies and in vitro techniques gave rise to the cholinergic hypothesis for $\mathrm{AD}$, and revealed $\mathrm{AD}$-associated decrease in choline acetyltransferase (ChAT) activity, and links with the presenilin 1, presenilin 2 and APOE-e4 genes, and vitamin B12/folate deficiency and high fat/high cholesterol diets. None relied on the use of NHPs or other animals.

NHP use can also cause direct human harm, in addition to delaying progress and diverting research funds from more relevant methods. The once much-vaunted AD 'vaccine' AN-1792 (AIP-001) dramatically slowed brain damage in an AD mouse model, and 'was well tolerated when tested in several animal species, including monkeys' in experiments prior to clinical trials (Sibal and Samson, 2001; Young, 2002). Despite the encouraging NHP data, clinical trials were suspended following CNS-inflammation and ischaemic strokes in 15 participants (Steinberg, 2002).

These situations have arisen because, quite simply but crucially, genes in the brains of humans and non-human primates, including chimpanzees and rhesus monkeys, differ in their levels of activity. These differences in brain activity between humans and monkeys can be traced right down to the molecular level, supporting 
the thesis that all animal species exhibit unique biological activities arising from their particular genetic make-up; and the smallest genetic difference or peculiarity can manifest as profound species differences. Interestingly, human genes exhibit greater protection against activity-related damage compared to monkey brains. This could help to explain why humans live longer than non-human primates and also why humans are more susceptible to age-related neurodegenerative diseases, such as Alzheimer's and Parkinson's (Emory University Health Sciences Center press release, 2003). Other major differences between humans and NHPs, particularly neurological differences, are now being elucidated that explain definitively why the latter can not serve as disease models for human beings. With regard to $\mathrm{AD}$, humans and great apes for example possess a particular type of projection neuron in the anterior cingulate cortex, which is known to be severely affected in the degenerative process of Alzheimer's disease (Nimchinsky et al., 1999). Also, the neuropeptide galanin that regulates cholinergic basal forebrain (CBF) function differs in its chemoanatomic organisation across species: in monkeys, all CBF neurons coexpress galanin, whereas in apes and humans galanin is found within a separate population of interneurons that are in close apposition to the CBF perikarya (Mufson et al., 1998). Because galaninergic fibres hyperinnervate CBF neurons in AD inhibiting acetylcholine release in the hippocampus, it may exacerbate cholinergic cellular dysfunction in AD. This difference could be critical when attempting to create an NHP AD model.

It is perhaps because of such evidence that many experts are now concluding that, in the words of Dr. John Xuereb, director of the Cambridge Brain Bank Laboratory, 'Alzheimer's, Parkinson's and other neurodegenerative diseases occur in humans and it is in human tissue that we will find the answers to these diseases' (BBC Radio Cambridge, UK, 2002).

- AIDS - NHPs do not develop AIDS when infected with HIV. Despite this fact, NHPs continue to be used in HIV/AIDS research, often with the 'related' simian immunodeficiency virus (SIV), or an artificial hybrid of the two (SHIV). As with so many artificially-induced animal models of human diseases, the differences are too profound to allow any resulting data from them to be extrapolated to humans (Kaufman et al., 1996). For example:

- Infection progresses at a different rate in SIV/SHIV infected monkeys compared to HIV-infected humans.

- Some proteins from the coat of the virus have vaccine-like effects in monkeys, but not in people.

- Chimpanzees have an alteration in their DNA that makes their immune systems detect and respond to HIV and SIV viruses differently (Anzai et al., 2003).

- The lymph nodes of HIV-infected people undergo structural changes and contain deposits of the virus; neither occurs in chimpanzees (Koopman et al., 1999). 
- Humans and chimpanzees have different ratios of crucial blood cells called T-lymphocytes.

- There are important differences in the cellular receptors involved in the infection process (Nath et al., 2000).

Because of these and many other differences, none of 30-plus vaccines (mostly tested in NHPs) has proved safe and effective in over 70 clinical trials. Recently, the 'Aidsvax'HIV vaccine failed to protect 8000 high-risk human volunteers despite proving efficacious in chimpanzees' (Wilson, 2003). In fact, some candidate antivirals screened using in vitro methods have gone directly into humans with little supportive in vivo data from prior animal experiments. One of the reasons is that there is no predictive animal model for HIV infection in humans (Wyand, 1992). Some scientists have acknowledged that even chimpanzees are unlikely to prove useful as animal models for understanding the mechanism of infection or means of treatment (Stott and Almond, 1995). Dr. Thomas Insel, as director of the Yerkes Primate Centre, admitted that 15 years of work in chimps has produced little data relevant to humans (Smaglik, 1999). The US Government, following a 10-year expert review, concluded that chimpanzees are a deficient model and redirected $\$ 10$ million of funding. Dr. Bolognesi of Duke University, USA, stated 'No animal models faithfully reproduce ... HIV infection and disease in humans, and the studies of experimental vaccines in animal models ... have yielded disparate results' (Bolognesi, 1994). In the opinion of a US-based group of researchers, 'the testing of vaccines and drugs in more animals will not be helpful if in the end these animals do not closely resemble humans. Even a vaccine that has $100 \%$ efficacy in [NHPs] ... might still be ineffective in humans. Conversely, a proficient vaccine developed in humans might never show benefit in the animal models' (Nath et al., 2000). Dr. Mark Feinberg, a leading AIDS researcher, summed it up thus: 'What good does it do you to test something [a vaccine] in a monkey? You find five or six years from now that it works in the monkey, and then you test it in humans and you realise that humans behave totally differently from monkeys, so you've wasted five years'.

In common with many other human diseases, everything we know about HIV and AIDS has been discovered without relying on animal models. Effective HIV protease-inhibitor and nucleoside-analogue drugs were conceived and developed using in vitro and in silico methods (DeVita et al., 1992; Mitsuya et al., 1985; Seelmeier et al., 1988; Soudeyns et al., 1991; Roberts et al., 1991; Vacca et al., 1994).

- Hepatitis - Hepatitis C virus (HCV) has infected hundreds of millions of people worldwide, and is a major cause of chronic liver disease. Chimpanzees have historically borne the brunt of $\mathrm{HCV}$ research, even though they respond to the virus very differently to humans. For example, significant and confounding differences have been reported in mother-infant transmission between humans and chimpanzees (Zanetti et al., 1995); chronic infection occurs much more frequently in humans (Major and Feinstone, 2000) humans progress to liver fibrosis and 
cirrhosis while chimpanzees do not, and humans suffer from hepatocellular carcinoma as a result of HCV though it is very rare in chimpanzees (Lanford et al., 2001). Naturally there are some similarities (in terms of liver response, for example), though the question must be asked (as with all animal models) if these similarities are sufficient to pursue relevant research that will lead to successful treatments.

In common with NHP models of many other human infectious diseases, the contribution of NHP experiments to treatment of HCV infection, especially with regard to vaccine development and understanding the mechanisms of hepatocellular damage, has been a failure (Rosen and Martin, 2000) with most of the progress made relying on in vitro and clinical studies. It must be stressed again that retrospective correlations between human-based knowledge and animal models are of no value. Now, with the recent breakthrough in the development of a successful in vitro culture system for the virus (Sun et al., 2004; Zhong et al., 2005), vaccine development is likely to accelerate as it did for other viruses such as polio and measles (Gallo, 1991).

Infectious disease research in NHPs or indeed, in any animals other than humans is almost guaranteed to fail because infectious agents are highly species specific. For instance, chimpanzees are essentially immune to the human AIDS and Hepatitis B and $\mathrm{C}$ viruses as detailed above, plus the malaria parasite and many other pathogens to which humans are susceptible. The Handbook of Animal Models of Infection, (Rouse and Wilson, 1999) observed: 'Up to this very day, all infectious diseases affecting humans are far from having appropriate animal models and, even in those cases where such infections are possible, the symptoms observed in animals and the course of the disease are often very different from those encountered in humans'. The recent anthrax attacks in the US mail were initially not taken seriously enough because experiments on monkeys showed the bacterium not to be fatal until 8-10 000 spores are inhaled. When people died from much smaller doses it became apparent that this does not apply to humans. Dr. Albert Sabin, inventor of the polio vaccine, swore under oath before the US Congress that: '... prevention [of polio] was long delayed by the erroneous conception of the nature of the human disease based on misleading experimental models of the disease in monkeys' (Sabin, 1984).

Space precludes an appraisal of the use of NHPs in multiple sclerosis, cancer, epilepsy, brain function and behavioural research, amongst many others. For the purposes of this review, it is sufficient to state that there is a similar pattern throughout all areas of NHP research: a lack of predictivity for humans, and a confounding dataset that often contrasts with what is subsequently discovered in humans.

\section{THE BIOLOGICAL BASIS OF HUMAN/NHP DIFFERENCES}

The differences between NHPs and human beings are clear to see, not just in the list of points made here, but of course more simplistically just by looking at individuals 
from all primate species. Depending on the methods of calculation, humans are 9799\% genetically identical to chimpanzees, our closest evolutionary relatives. Yet the physiological and biochemical differences that manifest due to this relatively small degree of genetic variation are immense (Enard et al., 2002; Hacia, 2001; Gagneux and Varki, 2001; Watanabe et al., 2004). On a superficial level we don't look alike or behave similarly; on a deeper level our biochemical differences mean that we suffer from different diseases, respond in different ways to infectious agents, have different metabolisms, and find different substances toxic. In short, an awful lot of subtle biochemical differences combine to make us very different indeed.

We are now learning why this is so, and it carries through to NHP use in biomedical research. A growing body of opinion, including many medical and scientific professionals, now believes that NHPs are just too different from human beings to serve as 'surrogate' humans in this way; they cannot be predictive of the human situation, and one cannot rely on data from NHP experiments when extrapolated to humans. NHP research can be manipulated in order to retrospectively correlate with and 'confirm' human data, but this should not be taken as evidence of its worth. The scientific and medical community at large can no longer ignore such a substantial body of information: when extrapolated to humans, NHP research has caused untold amounts of harm, a fraction of which is detailed here; directly by causing human suffering and death, and indirectly by delaying medical progress and diverting research funds from more appropriate methodology.

Those small genetic differences between us explain why this is the case. Some of these differences lie within the structural genes, in which small differences can completely change a gene's function and ability to do its job. Most of the differences, however, are now known to lie within the regulatory regions of our DNA; particular genes and sections of DNA that are involved in turning other genes 'on' and 'off' and modifying how their products do their jobs and interact with one another, in response to a variety of signals and stimuli. These can act highly promiscuously, and exert 'avalanche' effects upon hundreds of other genes. A small difference, therefore, can have far-reaching and extreme effects. For example, striking differences have been found in the levels of gene expression between humans and chimps in the brain and liver (Ruvolo, 2004).

\section{THE WAY FORWARD}

Many scientific techniques and methodologies are more relevant to human medicine and more predictive and reliable for human beings than NHP-oriented research, and are directly responsible for the great strides we are now making towards treating and curing the most widespread and debilitating human diseases. Batteries of humanbased tests provide reliable and relevant information on which to base further research and to speed the translation of research to the bedside.

These technologies include microarrays and other DNA technologies; proteomics and metabolomics; mathematical and computer modelling; epidemiology; human 
clinical research; myriad in vitro molecular biological techniques; microfluidics devices harbouring many types of human cells in an almost 'natural' environment and interacting with one another; and many more. Studies of brain function and neurological disorders account for much NHP research, yet the most dramatic differences between us and other primates are in the brain. Human brains can now be studied non-invasively using a huge array of imaging techniques such as positron emission tomography (PET), magnetoencephalography (MEG), magnetic resonance imaging (MRI) and functional magnetic resonance imaging (fMRI), transcranial magnetic stimulation (TMS), EROS (event-related optical signals), VBM (voxel-based morphometric analysis) and single photon emission computed tomography (SPECT). These enable the conscious brain to be observed while engaged in a variety of cognitive tasks (e.g. talking, singing, reading, and writing) of which NHPs are not even capable. Subtleties such as musical ability and memory skills can be identified, highlighting the redundancy of knowledge to be gained from NHPs. The Academy of Life Sciences at Aston University is an example of progress to be gained from multidisciplinary human clinical research.

Perhaps the most exciting technology with regard to drug development is 'microdosing', in which nanogram doses of new drugs are traced through the human body. Employed as 'Phase 0' clinical trials, microdosing provides extensive information about a drug's pharmacokinetic properties in a human environment and has already been endorsed by the FDA (April 2005) and the European Agency for the Evaluation of Medicinal Products (January 2003). The APC recommended that the use of microdosing in place of NHPs should be encouraged. Several companies exist whose mission is to develop and/or test drugs in an exclusively human context, such as Pharmagene, who observe; 'no animal species is sufficiently similar to man to act as a wholly reliable surrogate. Indeed there is extensive evidence that the use of animal (non-human) tissue can result in the generation of potentially misleading information' and Biopta, whose rationale is 'proof of concept in man'.

\section{TIME FOR OBJECTIVITY}

The limitations of nonhuman primate research and the risk of excessive faith in its results have not yet been widely appreciated by many. For a variety of political reasons, few scientists openly question whether primate models are a reliable research method. One of the main obstacles to open discussion and debate is that researchers, threatened by those who oppose their modus operandi, believe that best strategy is to defend all animal research regardless of its actual value (Gluck and Kubacki, 1991; Wiebers et al., 1994) and to discourage and actively suppress criticism from within their ranks (Arluke, 1994; Dunayer, 1993; Kaufman, 1994, 1991).

For the benefit of human medical progress, it is surely time for objectivity, transparency and honesty in the assessment of NHP models and their contribution to medical science. Only by ensuring this can we be confident that we are utilizing 
scientific technology to the full, performing the best translational research possible, and making real progress towards the relief of human suffering and disease.

\section{REFERENCES}

Animal Procedures Committee (2002). The use of primates under the Animals (Scientific Procedures) Act (1986). Analysis of current trends with particular reference to regulatory toxicology.

Anzai, T., et al. (2003). Comparative sequencing of human and chimpanzee MHC class I regions unveils insertions/deletions as the major path to genomic divergence, Proc. Natl. Acad. Sci. USA 100, 7708-7713.

Arluke, A. (1994). The ethical socialization of animal researchers, Lab. Animals (June), 30-35.

Bailey, J., Knight, A. and Balcombe, J. (2005). The future of teratology research is in vitro, Biogenic Amines 19, 97-145.

Benabid, A. L., Pollak, P., Louveau, A., Henry, S. and de Rougemont, J. (1987). Combined (thalamotomy and stimulation) stereotactic surgery of the VIM thalamic nucleus for bilateral Parkinson disease, Appl. Neurophysiol. 50, 344-346.

Benabid, A. L., Pollak, P., Hommel, M., Gaio, J. M., de Rougemont, J. and Perret, J. (1989). Treatment of Parkinson tremor by chronic stimulation of the ventral intermediate nucleus of the thalamus, Rev. Neurol. (Paris) 145, 320-323 (in French).

Bolognesi, D. P. (1994). A live-virus AIDS vaccine? J. NIH Res. 6, 59-62.

Boyd Group Papers (2002). The Use of Non-Human Primates in Research and Testing, http://www. boyd-group.demon.co.uk.

Brimblecombe, R. (1981). In: Risk-Benefit Analysis in Drug Research, Proceedings of an International Symposium Held at the University of Kent at Canterbury, England, 1980, Cavalla, J. F. (Ed.), p. 153. Society for Drug Research, Great Britain.

British Association for Tissue Banking website: http://www.batb.org.uk/

Caldwell, J. (1992). Species differences in metabolism and their toxicological significance, Toxicol. Lett. 64, 106.

Carson, S., Scheimbe, J., Mackars, A. and Vogin, E. E. (1971). Continuing toxicologic reassessment of isoprenaline aerosols, Pharmacologist 13, 272.

Chiba, M., et al. (2000). Comparative in vitro metabolism of indinavir in primates - a unique stereoselective hydroxylation in monkeys, Xenobiotica 30,117-129.

Conner, J. M. and Tuszynski, M. H. (2000), in: Central Nervous System Diseases: Innovative Animal Models from Lab to Clinic, Emerich, D. F., Dean III, R. L. and Sanberg, P. R. (Eds), p. 66. Humana Press, Totowa, New Jersey, USA.

DeVita, V. T. Jr., Hellman, S. and Rosenberg, S. A. (1992). AIDS Etiology, Diagnosis, Treatment, and Prevention, 3rd ed. J. B. Lippincott, Philadelphia, USA.

Druker, B. J. and Lydon, N. B. (2000). Lessons learned from the development of an Abl tyrosine kinase inhibitor for chronic myelogenous leukaemia, J. Clin. Invest. 105, 3-7.

Druker, B. J., Tamura, S., Buchdunger, E., et al. (1996). Effects of a selective inhibitor of the $\mathrm{Abl}$ tyrosine kinase on the growth of Bcr-Abl positive cells, Nature Med. 2, 561-566.

Dunayer, J. (2005). Censored: Faculty who oppose vivisection, Z Magazine (April) 57-60, www.zmag.org

Eason, C. T., et al. (1987). The effect of amrinone on platelets - cause and significance, Human Toxicol. 6, 436.

Eason, C. T., et al. (1990). The importance of pharmacokinetic and receptor studies in drug safety evaluation, Regulat. Toxicol. Pharmacol. 11, 288-307.

Emory University Health Sciences Center press release, 13 October 2003.

Enard, W., Khaitovich, P. and Klose, J., et al. (2002). Intra- and interspecific variation in primate gene expression patterns, Science 296, 340-343. 
European Agency for the Evaluation of Medicinal Products (EMEA) (2003). Position paper on nonclinical safety studies to support clinical trials with a single microdose. CPMP/SWP/2599/02.

Gagneux, P. and Varki, A. (2001). Genetic differences between humans and great apes, Molecular Phylogenetics and Evolution 18, 2-13.

Gallo, R. (1991). Virus Hunting. Basic Books, Harper Collins, London.

Geissler, J. F., Roesel, J. L., Meyer, T., et al. (1992). Benzopyranones and benzothiopyranones: a class of tyrosine protein kinase inhibitors with selectivity for the v-abl kinase, Cancer Research 52, 4492-4498.

Gluck, J. P. and Kubacki, S. R. (1991). Animals in biomedical research: the undermining effect of the rhetoric of the besieged, Ethics and Behavior 1, 157-173.

Gray, S. (2003). Breast cancer and hormone-replacement therapy: The Million Women Study, Lancet 362, 1332.

Greaves, P., Williams, A. and Eve, M. (2004). First dose of potential new medicines to humans: how animals help, Nature Reviews Drug Discovery 3, 226-236.

Guela, C., Wu, C.-K., Saroff, D., Lorenzo, A., Yuan, M. and Yankner, B. A. (1998). Nature Medicine 4, 827-831.

Hacia, J. G. (2001). Genome of the apes, Trends in Genetics 17, 637-645.

Hantraye, P. (1998). Modeling dopamine system dysfunction in experimental animals, Nuclear Med. Biol. 25, 721-728.

Harding, A. (2004). More compounds failing phase I. FDA chief warns that high drug attrition rate is pushing up the cost of drug development, The Scientist (6 August).

Heywood, R. (1990). Clinical toxicity — could it have been predicted? In: Animal Toxicity Studies: Their Relevance for Man, Lumley, C. E. and Walker, S. R. (Eds), pp. 57-67. Quay, Lancaster, UK.

Hodgson, J. (2001). ADMET — turning chemicals into drugs, Nature Biotechnol. 19, 722-726.

Home Office Primates Stakeholder Forum (2004): Rapporteur's Report. Website: http://www. homeoffice.gov.uk/docs4/apc_non_human_primates.pdf

House of Lords Select Committee on Animals in Scientific Procedures (2002). HL150-I, 16 July.

Huang, J., et al. (2000). A modified transorbital baboon model of reperfused stroke, Stroke 31, 3054 3063.

Kaufman, S. R. (1991). UR researchers refused to debate, Democrat and Chronicle, Rochester, NY, July 19.

Kaufman, S. R. (1994). Animal protection and medical science [letter], Lancet 343, 1574.

Kaufman, S. R., Cohen, M. J. and Simmons, S. (1996). In: Shortcomings of AIDSRelated Animal Experimentation. Medical Research Modernization Committee, New York [www.mrmcmed.org/aids.html].

Koopman, G., et al. (1999). The relative resistance of HIV type 1-infected chimpanzees to AIDS correlates with the maintenance of follicular architecture and the absence of infiltration by CD8+ cytotoxic T lymphocytes, AIDS Res. Hum. Retroviruses 15, 365-373.

Lanford, R. E., Bigger, C., Bassett, S. and Kimpel, G. (2001). The chimpanzee model of hepatitis C virus infections, ILAR Journal 42, 117-126.

Lazarou, J., Pomeranz, B. H. and Corey, P. N. (1998). Incidence of adverse drug reactions in hospitalized patients: A meta-analysis of prospective studies, J. Amer. Med. Assocn. 279, 1200 1205.

Lindholm, D. (1997). Models to study the role of neurotrophic factors in neurodegeneration, J. Neural Transm. Suppl. 49, 33-42.

Lumley, C. E. and Walker, S. R. (1990). In: Animal Toxicity Studies: Their Relevance for Man, p. 73. Quay, Lancaster, UK.

Lydon, N. B., Adams, B., Poschet, J. F., et al. (1990). An E. coli expression system for the rapid purification and characterization of a $v$-abl tyrosine protein kinase, Oncogene Research 5, 161173. 
Major, M. E. and Feinstone, S. M. (2000). Characteristics of hepatitic C infectious clones in chimpanzees, Curr. Topics Microbiol. Immunol. 242, 279-298.

Mann, R. D. (1984), Modern Drug Use: An Enquiry on Historical Principles. MTP Press, Lancaster, UK.

Marshall, E. (2000). Gene thereapy on trial, Science 288, 951-957.

Mitsuya, H., Weinhold, K. J., Furman, P. A., et al. (1985). 3'-Azido-3' deoxythymidine (BW A509U): an antiviral agent that inhibits the infectivity and cytopathic effect of human T-lymphotropic virus type III/lymphadenopathy-associated virus in vitro, Proc. Natl Acad. Sci. USA 82, 7096-7100.

Mufson, E. J., Kahl, U., Bowser, R., Mash, D. C., Kordower, J. H. and Deecher, D. C. (1998). Galanin expression within the basal forebrain in Alzheimer's disease. Comments on therapeutic potential, Ann. N Y Acad. Sci. 863, 291-304.

Nath, B. M., et al. (2000). The chimpanzee and other non-human-primate models in HIV-1 vaccine research, Trends Microbiol. 8, 426-431.

Neff, S. (1989). Clinical relevance of stroke models, Stroke 20, 699-701.

NHP Study website: http://www.nhpstudy.com/call_for_evidence.pdf

Nimchinsky, E. A., Gilissen, E., Allman, J. M., Perl, D. P., Erwin, J. M. and Hof, P. R. (1999). A neuronal morphologic type unique to humans and great apes, Proc. Natl Acad. Sci. USA 96, 52685273.

Nuffield Council on Bioethics (2005). The Ethics of Research Involving Animals, http://www. nuffieldbioethics.org.

Olson, H., et al. (2000). Concordance of the toxicity of pharmaceuticals in humans and animals, Regul. Toxicol. Pharmacol. 32, 56-67.

Palfreyman, M. G., Charles, V. and Blander, J. (2002). The importance of using human-based models in gene and drug discovery, Drug Discovery World, 33-40, [Personal communication from professional statistician].

Pharmagene website: http://www.pharmagene.com/ (Active programmes).

Roberts, N. A., Martin, J. A., Kinchington, D., et al. (1990). Rational design of peptide-based HIV proteinase inhibitors, Science 248, 358-361.

Rosenk, H. R. and Martin, P. (2000). Hepatitis B and C in the liver transplant recipient, Seminars Liver Dis. 20, 465-480.

Rouse, M. S. and Wilson, W. R. (1999). General methodologies for animal models, in: Handbook of Animal Models of Infection, p. 9. Academic Press.

Ruvolo, M. (2004). Comparative primate genomics: the year of the chimpanzee, Curr. Opin. Genet. Dev. 14, 650-656.

Sabin, A. (1984). Statement before the Subcommittee on Hospitals and Health Care, Committee on Veterans Affairs, House of Representatives, April 26, serial no. 98-48.

Schardein, J. L. (1993). Chemically Induced Birth Defects, 2nd edn. Marcel Dekker, New York.

Schein, P. S., Davis, R. D., Carter, S., Newman, J., Schein, D. R. and Rall, D. P. (1970). The evaluation of anticancer drugs in dogs and monkeys for the prediction of qualitative toxicities in man, Clin. Pharmacol. Ther. 11, 3-40.

Seelmeier, S., et al. (1988). Human immunodeficiency virus has an aspartic-type protease that can be inhibited by pepstatin A, Proc. Natl Acad. Sci, USA 85, 6612-6616.

Sibal, L. R. and Samson, K. J. (2001). Nonhuman primates: A critical role in current disease research, ILAR Journal 42, 74-84.

Smaglik, P. (1999). AIDS vaccine researchers turn from chimps to monkeys, The Scientist 13, 7, August 16th.

Smith, D., Trennery, P., Farningham, D. and Klapwijk, J. (2001). The selection of marmoset monkeys, Callithrix jacchus, in pharmaceutical toxicology, Laboratory Animals 35, 117-130.

Snowdon, D. A., Greiner, L. H., Mortimer, J. A., Riley, K. P., Greiner, P. A. and Markesbery, W. R. (1997). Brain infarction and the clinical expression of Alzheimer disease. The Nun Study, J. Amer. Med. Assocn. 277, 813-817. 
Soudeyns, H., Yao, X.-J., Gao, Q., et al. (1991). Anti-human immunodeficiency virus type 1) activity and in vitro toxicity of $2^{\prime}$-deoxy-3'-thiacytidine $(\mathrm{BCH} 189)$, a novel heterocyclic nucleoside analog, Antimicrobial Agents and Chemotherapeutics 35, 1386-1390.

Spriet-Pourra, C. and Auriche, M. (1994). Drug Withdrawal from Sale, 2nd edn. Scrip Reports, PJB Publications, New York, USA.

St. George-Hyslop, P. H. and Westaway, D. A. (1999). Alzheimer's disease. Antibody clears senile plaques, Nature 400, 116-117.

Steinberg, D. (2002). Companies halt first Alzheimer vaccine trial, The Scientist 16, 22, April 1st.

Stott, J. and Almond, N. (1995). Assessing animal models of AIDS, Nature Medicine 1, 295-297.

Sun, B. S., Pan, J., Clayton, M. M., Liu, J., Yan, X., Matskevich, A. A., Strayer, D. S., Gerber, M. and Feitelson, M. A. (2004). Hepatitis C virus replication in stably transfected HepG2 cells promotes hepatocellular growth and tumorigenesis, J. Cell Physiol. 201, 447-458.

UK Human Tissue Bank website: http://www.ukhtb.org

US Food and Drug Administration website: http://www.fda.gov/oc/initiatives/criticalpath/whitepaper. html

Vacca, J. P., et al. (1994). L-735,524: an orally bioavailable human immunodeficiency virus type 1 protease inhibitor, Proc. Natl. Acad. Sci, USA 91, 4096-4011.

Watanabe, H., et al. (2004). DNA sequence and comparative analysis of chimpanzee chromosome 22, Nature 429, 382-388.

Wiebers, D. O., Leaning, J. and White, R. D. (1994). Animal protection and medical science, Lancet 343, 902-904.

Wiebers, D. O., Adams, H. P. Jr. and Whisnant, J. P. (1990a). Animal models of stroke: are they relevant to human disease? Stroke 21, 1-3.

Wiebers, D. O., Whisnant, J. P., Sandok, B. A. and O'Fallon, W. M. (1990b). Prospective comparison of a cohort with asymptomatic carotid bruit and a population-based cohort without carotid bruit, Stroke 21, 984-988.

Wilson, C. (2003). HIV vaccine hopes still high, New Scientist 2385, 8 March.

Wyand, M. S. (1992). The use of SIV-infected rhesus monkeys for the preclinical evaluation of AIDS drugs and vaccines, AIDS Res. Human Retroviruses 8, 349-356.

Xceleron website: http://www.xceleron.co.uk/metadot/index.pl?id=2224\&isa=Newsitem\&op=show

Young, E. (2002). Alzheimer's vaccine trial suspended, The New Scientist, 22 January.

Zanetti, A. R., Tanzi, E., Paccagnini, S., Principi, N., Pizzocolo, G., Caccamo, M. L., D’Amico, E., Cambie, G. and Vecchi, L. (1995). Mother-to-infant transmission of hepatitis C virus. Lombardy Study Group on Vertical HCV Transmission, Lancet 345, 289-291.

Zhong, J., Gastaminza, P., Cheng, G., Kapadia, S., Kato, T., Burton, D. R., Wieland, S. F., Uprichard, S. L., Wakita, T. and Chisari, F. V. (2005). Robust hepatitis C virus infection in vitro, Proc. Nat. Acad. Sci. USA 102, 9294-9299. 